\title{
Über die Wirkungsweise der Gefrierkerne im unterkühlten Wasser
}

\author{
Von Walter RaU \\ Aus der Forschungsstelle für Physik der Stratosphäre in der Max-Planck-Gesellschaft, Weißenau \\ (Z. Naturforschg. 5 a, 667-675 [1950]; eingegangen am 10. November 1950)
}

\begin{abstract}
In Laboratoriumsversuchen an unterkühlten Wassertropfen wird das Spektrum der Gefrierkerne im Temperaturbereich bis $-20^{\circ} \mathrm{C}$ untersucht. Die beiden Maxima um $-4^{\circ} \mathrm{C}$ und - $12^{\circ} \mathrm{C}$ können an einem großen Beobachtungsmaterial bestätigt werden. Eine künstliche Beeinflussung des Spektrums ist möglich: durch Zugabe von gepulverten Substanzen ist zu erreichen, daß das erste Maximum verstärkt wird, und zwar auf Kosten des zweiten Maximums. Das erste Maximum wird durch frische Kerne hervorgerufen, welche die Eisbildung beim Eindringen in die unterkühlten Tropfen auslösen. Die Struktur der Kerne zeigt dabei keinen Einfluß; notwendig ist aber, daß die Kerne trocken sind. Im Tropfeninnern befindliche Kerne lösen das Erstarren offenbar frühestens im zweiten Maximum aus. Diese Wirkungsweise der Kerne wird damit gedeutet, daß die an trockenen Kernen beim Eindringen in Wasser entstehende elektrische Doppelschicht im unterkühlten Wasser die Kristallisation auslöst.

Die Versuche ergeben eine zwanglose Erklärung für die Beobachtungen über die atmosphärische Eisbildung, welche nach $\mathrm{Peppler}$ vorwiegend bei $-12^{\circ} \mathrm{C}$ einsetzt, nach neueren Beobachtungen von Schwerdtfeger bei der Schauerbildung über See aber auch schon überwiegend bei $-4^{\circ} \mathrm{C}$ vorkommen kann.
\end{abstract}

$D^{i}$ ie Theorie der Phasenbildung ${ }^{1}$ läßt erwarten, $\mathrm{da}$ in der Natur die Eisbildung im allgemeinen unter Mitwirkung von Kernen erfolgt. Trotz der dafür erbrachten experimentellen Bestätigungen ist die Frage nach den spezifischen Eigenschaften dieser Kerne noch nicht eindeutig beantwortet. Am günstigsten müßten natürlich mit Eis isomorphe Substanzen sein. Wie aber u. a. W e i c k m a n ${ }^{2}$ ausgeführt hat, ist es äußerst schwierig, solche Substanzen bei Eis überhaupt anzugeben. Besonders umstritten wurde immer wieder die infolge Isomorphie vermutete Ausnahmestellung von kristallinem Quarz, der bei seinem reichen Vorkommen mindestens auf dem Festland überall zur Eisbildung zur Verfügung wäre. Aber schon $\mathrm{T}$ a m mann und $\mathrm{B} \ddot{\mathrm{c}} \mathrm{chner}{ }^{3}$ haben zeigen können, daß Quarz keineswegs bevorzugt ist. In ihren Versuchen konnte der Gefriereinsatz von reinem Wasser in Glasröhrchen von $-15,4$ bis $-20^{\circ} \mathrm{C}$ durch kristallinen Quarz auf $-8,8$ bis $-12,5^{\circ} \mathrm{C}$ gehoben werden, durch amorphen Quarz aber auch auf $-9,0$ bis $-13,5^{\circ} \mathrm{C}$, ja durch Elektrolyteisen sogar auf $-7,7$ bis $-11,5^{\circ} \mathrm{C}$. Dagegen ergab sich nach $\mathrm{S} \mathrm{chulz}{ }^{4}$ bei der Fortführung dieser Versuche durch

1 Vgl. M. V o $1 \mathrm{~m}$ e r, Kinetik der Phasenbildung, Dresden u. Leipzig 1939, S. $204 \mathrm{ff}$.

$2 \mathrm{H}$. Wei ckmann, Ber. Deutsch. Wetterdienstes US-Zone Nr. 6 [1949].

3 G. T a m m a n n u. A. B ü chner, Z. anorg. allg. Chem. 222, 371 [1935].
Findeisen und Schulz mit dem Ziele, geeignete "Sublimationskerne“ zu finden, wieder eine bevorzugte Wirkung der kristallinen Quarzmodifikationen. Die angegebenen Gefriereinsätze passen aber recht gut zu den Ergebnissen von Tammann, besonders wenn man noch in Betracht zieht, daß bei Schulz auch offensichtlich strukturfremde Stoffe, wie Bimsstein, Meteoreisen, Zinnober u. a., das Gefrieren schon bei -4 bis $-5^{\circ} \mathrm{C}$ auslösen konnten. Auch die neuerdings gefundene gute Wirksamkeit der in der Atmosphäre wohl selten vorkommenden Stoffe, wie AgJ, $\mathrm{PbJ}_{2}$ u. a., ist offenbar von gewissen Nebenbedingungen abhängig und nicht allein der Isomorphie oder Strukturähnlichkeit zuzuschreiben ${ }^{5}$.

Die Wirkung einzelner individueller Gefrierkerne bei charakteristischen Temperaturen hatte der Verf. in früheren Versuchen nachweisen können ${ }^{6}$. Zu beobachten waren dabei aber immer nur die auswachsenden Eiskeime, nie die Kerne selbst. Deren Aktivität hing aber sehr von ihrer Vorgeschichte ab: die Kerne altern, werden durch Abtrocknen aber auch wieder aktiv. Die Frage nach der Natur der Kerne blieb wieder offen. Es gelang aber, ein Gefrierkernspektrum, d.h. eine Verteilungskurve der charakteristischen Temperaturen der Gefrierkerne, statistisch

4 G. S chulz, Meteor. Rundschau 1, 237 [1948].

5 B. Vonnegut, J. appl. Physics 18, 593 [1947].

6 W. R a u, Schr. Deutsch. Akad. Luftfahrtforschg. 8, 65 [1944]. 
zu ermitteln (Kurve 1, Abb. 1). Dessen Maxima bei $-4^{\circ},-10^{\circ}$ bis $-12^{\circ}$ und $-20^{\circ} \mathrm{C}$ müßten dann wohl von verschiedenen Kernarten oder wenigstens Kernwirkungen herrühren, so daß eine eventuelle Beeinflussung dieses Spektrums durch gepulverte Substanzen oder dergl. genaueren Aufschluß über die natürlichen Kerne selbst geben müßte.

Über eine derartige Einwirkung auf das natürliche Gefrierkernspektrum und die Möglichkeit, die atmosphärische Eisteilchenbildung damit befriedigend $\mathrm{zu}$ erklären, ist schon berichtet worden ${ }^{7}$. Die damals angekündigte Veröffentlichung der gesamten Versuchsergebnisse kann wegen äußerer Umstände erst heute erfolgen.

\section{Die Untersuchungsmethode}

Das oben erwähnte Gefrierkernspektrum (Kurve 1, Abb. 1) war aus 2 Versuchsreihen mit je 12 Tropfen bei zehnmal wiederholter Kühlung und dazwischenliegendem Auftauen ermittelt worden. Die Wassertropfen lagen dabei auf polierten Chromflächen in einer abgeschlossenen Kältekammer. Diese Versuchsanordnung wurde beibehalten, bot sie doch den Vorteil, die zu untersuchenden Kernsubstanzen in Wassertropfen zu bringen, deren Gefriereinsatz unmittelbar vorher bestimmt worden war. Um auch den Abtrocknungseffekt näher untersuchen zu könren, wurde ein kleiner, elektrisch beheizbarer Behälter in die Kammer eingebaut, dessen dicht schließender Deckel durch einen Elektromagneten abgehoben werden konnte, worauf der Luftstrom des zur Erzielung gleichmäßiger Temperaturverteilung in der Kammer befindlichen Ventilators die Versuchssubstanzen aus dem Behälter heraus auf die Spiegel und in die Wassertropfen tragen konnte. Die Kammer blieb also während der Versuche geschlossen; eine frische Kernzufuhr aus der freien Zimmerluft war ausgeschlossen. Diese Bedingung war bei den Untersuchungen anderer Autoren, bei denen die Kernsubstanzen in Glasröhrchen gegeben wurden, sicher nie streng erfüllt.

II. Das allgemeine Gefrierkerns pektrum

Da bei unserer Versuchsfolge jede Kühlung der Tropfen mit Zugabe von Kernsubstanzen immer in Kontrollversuche ohne Kernzugabe eingeschlossen wurde, bzw. die sich ergebende „künstliche“ Beeinflussung des Gefrierkernspektrums nicht bei allen darauf abzielenden Versuchen gelang, überwogen die Versuche ohne Zusatz; es wird daher zunächst einmal die Zusammenfassung sämtlicher neuen Versuchsserien gegeben, da damit der allgemeinst mögliche Kerngehalt erfaßt wird. Unsere Versuchsergebnisse mit absichtlicher Fremdsubstanzbeigabe können dann als

7 W. R a u, Wetter u. Klima 1, 81 [1949]. die besonderen Abweichungen von diesem allgemeinen Gefrierkernspektrum beschrieben werden.

Die Kurve 2 (Abb. 1) zeigt das Gefrierkernspektrum, ermittelt aus 24 neuen Versuchsreihen mit je 12 Tropfen (je 0,6 $\mathrm{cm}^{3}$ ) auf den Spiegeln und je nach besonderem Zweck der Reihe wechselnder Anzahl

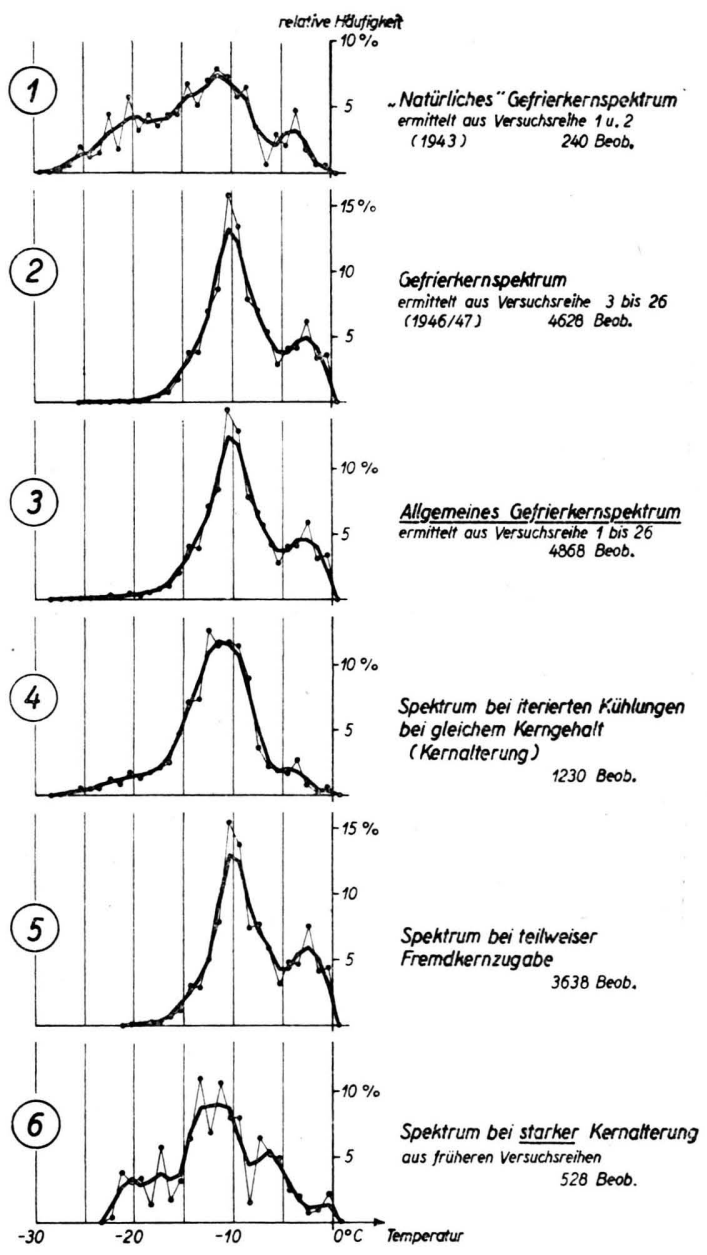

Abb. 1. Gefrierkernspektrum bei verschiedenem Kerngehalt. [Stark ausgezogene Kurven sind $(1+2+1)$-gemittelt.]

von Kühlungen. Damit die einzelnen Kurven leichter miteinander verglichen werden können, sind als Ordinaten die relativen Häufigkeiten eingetragen. Bei allen Zusammenfassungen mehrerer Versuchsreihen sind aber nur absolute Häufigkeiten verwendet, so daß also dort die Beobachtungen immer in der direkten, experimentell ermittelten Häufigkeit eingehen ${ }^{8}$.

$8 \mathrm{Um}$ ein möglichst allgemeines Ergebnis zu erhalten, wurden sämtliche ausgeführten Versuchsreihen ohne Auswahl berücksichtigt; ausgelassen wurden nur Versuche, bei denen die Tropfen gelöste Substanzen enthielten. 
Ein Vergleich mit der Kurve 1 zeigt, daß die neuen Beobachtungsreihen das früher mitgeteilte Gefrierkernspektrum voll bestätigen. Die Maxima liegen wieder bei $-4^{\circ} \mathrm{C}$ und bei -10 bis $-12^{\circ} \mathrm{C}$. Wegen der wesentlich geringeren Anzahl Einzelbeobachtungen hat man bei Kurve 1 eine stärkere statistische Streuung. Der steilere Verlauf unterhalb $-12^{\circ} \mathrm{C}$ rührt von der verschieden starken Mitwirkung der Kernalterung her, worauf später noch eingegangen wird.

Aus unserem gesamten Beobachtungsmaterial kann somit durch Zusammenfassung dieser beiden Kurven zur Kurve 3 ein ganz allgemeines Gefrierkernspektrum abgeleitet werden; darin sind 4868 Einzelbeobachtungen bei verschiedenstem Kerngehalt enthalten.

Unterteilt man das Beobachtungsmaterial in zwei Gruppen, von denen die eine nur Kühlungen um$\mathrm{fa} \mathrm{t}$, die bei gleichbleibendem Gesamtkerngehalt wiederholt wurden (normale Alterung), während in die zweite Gruppe alle Versuchsreihen mit wiederholter Beimengung frischer Kerne kommen, so wird die Kurve 3 zerlegt in die Kurven 4 und 5 (Abb. 1). Der Unterschied kommt schon ganz deutlich heraus: Bei der Alterung der Gefrierkerne (Kurve 4) verschwindet das erste Maximum fast ganz, das zweite Maximum wird ausgeprägter, aber flacher, und reicht einige Grade tiefer. Bei teilweise neuen Gefrierkernen (Kurve 5) wird dagegen das erste Maximum um $-4^{\circ} \mathrm{C}$ stark ausgeprägt und das Hauptmaximum ist sehr eng auf $-10^{\circ} \mathrm{C}$ zusammengedrängt; die ganze Kurve reicht nur bis $-20^{\circ} \mathrm{C}$.

Der Vollständigkeit halber zeigen wir noch in Kurve 6 (Abb. 1) ein Spektrum, das wir noch nachträglich aus früheren Untersuchungen errechnet haben ${ }^{9}$. Damals lag das Hauptaugenmerk auf der Kernalterung, um möglichst tiefe Unterkühlungen zu bekommen. Anfangskühlungen blieben meist unberücksichtigt; der Verlauf ist weniger ausgeprägt. Auch hier ist das zweite Maximum flacher und reicht zu tieferen Temperaturen; das schwache Maximum um $-20^{\circ} \mathrm{C}$ kommt ebenfalls wieder vor, im Gegensatz zu unseren neueren Untersuchungen, wo meist keine längere Alterung abgewartet worden war; man sieht, wie dadurch der Kurvenverlauf bei diesen tieferen Temperaturen immer deutlich beeinflußt wird. Eine längere Kernalterung war in unseren neueren Versuchen schon deswegen nicht mehr möglich, weil die Tropfen bei mehrmaliger Zugabe von Fremdkernen schließlich so viel Verunreinigungen enthielten, daß schon

9 Diese Versuchsreihen sind in der vorliegenden Arbeit nicht statistisch verwertet. der bereits erwähnte Effekt der Abtrocknung der Kerne am Tropfenrand und die damit verknüpfte verfrühte Eisbildung die tiefen Unterkühlungen ausschloß.

\section{Die künstliche Beeinflussung des Gefrierkernspektrums}

Nachdem im vorhergehenden Abschnitt die Wirkung von absichtlich eingebrachten Fremdsubstanzen auf die Unterkühlbarkeit im summarischen Effekt gezeigt wurde, soll nun diese Einwirkung im einzelnen beschrieben werden.

Bei diesen Versuchen wurde immer zuerst der Gefrierpunkt der reinen Wassertropfen (destilliertes Wasser) bestimmt. Lag er nicht bei allen gleichzeitig in der Kammer befindlichen Tropfen eindeutig unter $-10^{\circ} \mathrm{C}$, so wurde wieder aufgetaut und erneut gekühlt. Erst wenn alle Tropfen sicher unter $-10^{\circ} \mathrm{C}$ erstarrten, wurden frische Kerne zugegeben. In Anlehnung an die eingangs erwähnten Versuche von Tammann wurden die zu untersuchenden, meist in einer Reibschale gepulverten Substanzen anfangs einfach bei abgenommenem Kammerdeckel in die Tropfen gebracht. Ein durchgehender Erfolg blieb dabei aus; die Eisbildung kam, von seltenen Ausnahmen abgesehen, trotzdem wieder bei der zuvor ermittelten Temperatur. Dabei konnte beobachtet werden, wie die Substanzen beim Aufstreuen zum großen Teil gar nicht in die Tropfen hineingelangten, sondern auf der Oberfläche schwammen und darauf zum Rand abrutschten, ohne daß sich die Tropfen darum gekümmert hätten. Größere Körner konnten sogar durch unterkühlte Tropfen hindurchsinken, ohne Erstarrung auszulösen.

Ein gewisser Erfolg war erst dadurch zu erzielen, daß bei abgenommenem Kammerdeckel Staub von einem abgeschalteten elektrischen Heizofen weg in die Kammer geblasen wurde. In vielen Fällen trat dann die Kristallisation bei merklich höheren Temperaturen auf; insgesamt war der allgemeine Kerngehalt in unserem damals ungeheizten Arbeitsraum im Temperaturbereich oberhalb $-10^{\circ} \mathrm{C}$ wenig wirksam.

Ganz anders wurde es erst, als der in Abschnitt I beschriebene Kernbehälter in Verwendung kam. Jetzt setzte die Eisbildung in den unterkühlten Wassertropfen sofort ein, sobald die fein zerriebenen Substanzen aus dem Behälterchen herausgewirbelt wurden. Während man sonst bei den Unterkühlungsversuchen immer lange warten mußte, bis schließlich die Tropfen so zufällig nacheinander gefroren - die be- 
kannte Impfung mit Eiskristallen natürlich ausgenommen - konnte jetzt die Eisbildung gewissermaßen für alle Tropfen „eingeschaltet“ werden. Hauptbedingung war nur, daß die Substanzen vorher gut beheizt, also trocken waren. Ohne Vorheizung trat die Gefrierauslösung oft nur zögernd, wenn überhaupt auf.

Wieder fiel ein großer Teil der aufgewirbelten Substanzen wirkungslos auf die Tropfen. Offenbar bildeten nur wenige, im Mikroskop nicht mehr sichtbare Teilchen Eis; an deren vermutlichen Auftreffstellen schwammen dann rasch auswachsende Nadelbündel auf der Tropfenoberfläche - häufig mehrere Eiskeime zugleich in einem einzigen Tropfen - von wo aus dann die ganzen Tropfen in Eis verwandelt wurden. Eine Beschränkung auf nur einen Eiskeim pro Tropfen, wie wir es in früheren Versuchen immer bei der höchsten charakteristischen Temperatur beobachteten, ist hier selbstverständlich nicht mehr eingehalten.

Wir möchten noch erwähnen, daß der normale Gefrierpunkt unserer Wassertropfen immer bei $0^{\circ} \mathrm{C}$ lag und daß wir ihn zwischendurch zur Kontrolle unserer Temperaturangaben bestimmten, indem wir die Tropfen wieder kühlten, solange noch nicht alles Eis abgeschmolzen war. Von Eisresten aus begann das Gefrieren immer ganz knapp unter $0^{\circ} \mathrm{C}$. Die beobachteten Unterkühlungen können somit nicht von einer schädlichen Verunreinigung der Tropfen oder der Kammer herrühren.

\section{Die untersuchten Substanzen}

Die Frage, ob gewisse, evtl. mit Eis „isomorphe“ Substanzen als Gefrierkerne besonders geeignet sind, ist in den Kurven der Abb. 2 und 3 beantwortet. Obgleich schon, wie erwähnt, das Einzelexperiment die Wirkung der einzelnen Substanzen erkennen ließ, haben wir doch die Darstellung mit der spektralen Verteilung beibehalten, weil darin erst die volle Reichweite der Beeinflussung zum Ausdruck kommt und weil die bloße Angabe von erzielten Grenz- oder gar Durchschnittstemperaturen das Wesentliche nicht erkennen läßt.

Die Kurven der Abb. 2 zeigen den Effekt von verschiedenen kristallinen Substanzen bzw. Substanzen mit wahrscheinlich kristallinem Anteil: sie haben alle dieselbe Wirkung. Die Eisbildung erfolgt im ersten Maximum, das vor allem in der Summenkurve 13 für alle Versuche mit kristallinem Staub bei stark reduzierter statistischer Streuung ganz klar herauskommt. Das zweite Maximum ist nahezu unbesetzt; die wenigen Fälle der Eisbildung in diesem Bereich rühren

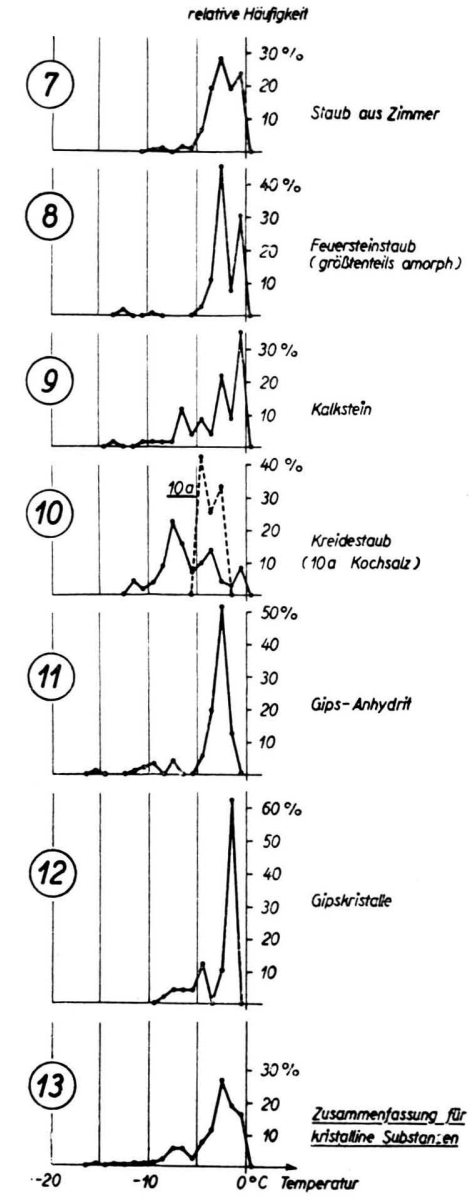

Abb. 2. Gefrierauslösung durch (teilweise) kristalline Substanzen.

davon her, daß mitunter zu wenig Substanz aufgewirbelt wurde, so daß einzelne Tropfen, vor allem die, welche etwas außerhalb der Luftströmung lagen, nicht getroffen wurden und dann meistens bei ihrem zuvor bestimmten Erstarrungspunkt ab $-10^{\circ} \mathrm{C}$ wiederkamen.

Die gute Wirkung kann aber aus der speziellen Kristallstruktur heraus nicht erklärt werden: Wenn auch Staub aus dem Zimmer (Kurve 7) und Gesteinsstaub (Kurve 8 und 9) gewisse Bestandteile von Quarz oder Ähnlichem enthalten könnten, so trifft dies bei Gips (monoklin, Kurven 11 und 12) und Kochsalz $^{10}$ (kubisch, Kurve 10 a) bestimmt nicht zu. Dabei wäre auch nicht zu verstehen, weshalb die Trocknung der Substanzen im Kernbehälter die Wirkung so eindeutig erhöhte, in deutlicher Parallele zu unserem schon erwähnten Abtrocknungseffekt. Denn

10 Die Kühlungen bei Verwendung von $\mathrm{NaCl}$ sind in unserer Statistik weggelassen. 


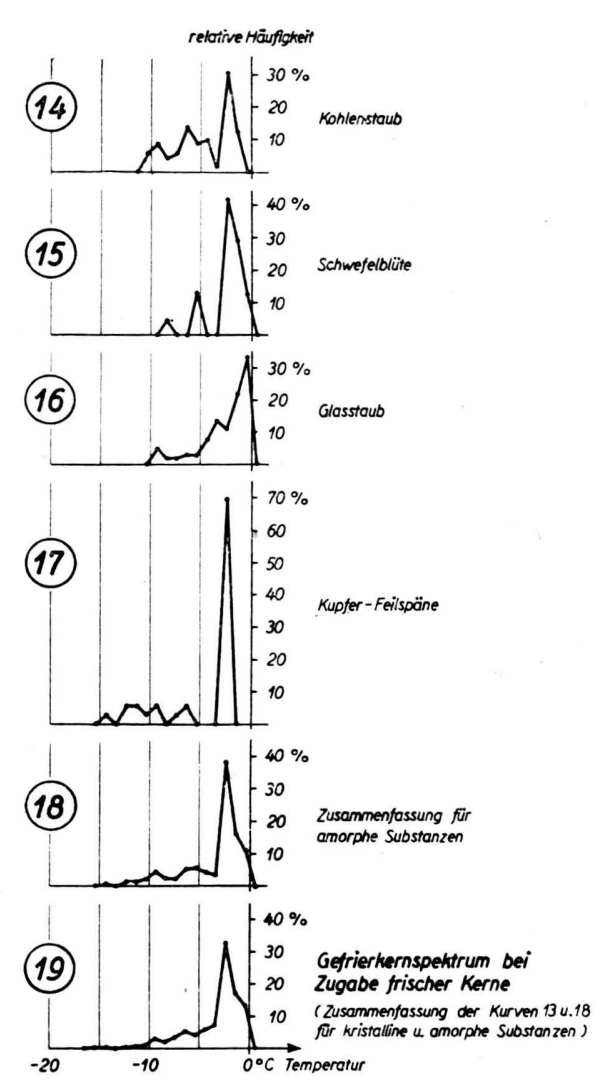

Abb. 3. Gefrierauslösung durch amorphe Substanzen.

Staub aus einem geheizten, trockenen Raum löste die Eisbildung sofort aus; war er einem ungeheizten (feuchteren) Zimmer entnommen, blieb er ohne Wirkung; im Kernbehälter zusätzlich getrocknet, bekam er aber dieselbe gute Wirkung ${ }^{11}$. Nur Kreidestaub (Kurve 10) war trotz Trocknung weniger günstig; das rührt aber wohl daher, daß er leicht verklebt und sich dann schlecht aufwirbeln läßt.

$\mathrm{Da}$ die Kristallstruktur überhaupt nicht wesentlich ist, sieht man auch daran, daß die amorphen Substanzen der Abb. 3 dasselbe Ergebnis bringen. Ja, die Wirkung ist auch nicht auf dielektrische Stoffe beschränkt, sondern kann auch mit ganz feinem $\mathrm{Cu}$-, Messing-, Al- und Fe-Staub (z. B. Kurve 17) erzielt werden. Es kann sich also auch nicht um Staubteilchen mit einer elektrischen Ladung handeln, die bei di-

11 Einen Unterschied zwischen den Kernen aus geheizten und ungeheizten Räumen fand auch $\mathrm{H}$. W e i ckm a n n, Forsch.-Ber. 1730 Zentr. wiss. Ber. Wes. Luftfahrtforschg. (ZWB) [1942], führte ihn aber auf die bevorzugte Wirkung von Verbrennungsprodukten der Ofenheizung zurück. Solche sind aber in unseren Räumen bei Zentralheizung nicht vorhanden gewesen. elektrischen Stoffen bevorzugt auftreten müßte. Versuche, durch Anlegen eines elektrischen Feldes zwischen Spiegel und einem darübergespannten Drahtnetz evtl. geladene Teilchen am Erreichen der Tropfen zu hindern, verliefen negativ. - Ein Unterschied zwischen amorphen Substanzen (Kurve 18) und kristallinen (Kurve 13) ist überhaupt nicht zu erkennen.

Wenn man also nur frische, gut getrocknete Kerne verwendet, bzw. in der Natur im Einzelfall nur solche zur Wirkung gelangen können (vgl. Abschn. VII), so ist das Spektrum der Kurve 19 zu erwarten: die beabsichtigte Beeinflussung des früher mitgeteilten „natürlichen“ Spektrums der Gefrierkerne ist somit vollständig gelungen ${ }^{12}$.

V. Das Gefrierkernspektrum im Wechselspiel zwischen Kernalterung und frischer Kernzufuhr

Da praktisch alle Substanzen in unseren Versuchen die gleiche Wirkung zeigten, müssen bestimmte gemeinsame Eigenschaften die spezifische Wirkungsweise der Gefrierkerne verursachen, wenigstens im Bereich des ersten Maximums. Wir haben nun versucht, uns einen Einblick in die verwickelten Verhältnisse zu verschaffen, indem wir gewisse Nachwirkungen untersucht haben. Dazu haben wir die Kühlungen nach bestimmten Gesichtspunkten zu Gruppen zusammengefaßt und dafür die spektralen Verteilungen ermittelt.

1. Beim früheren Spektrum (Kurve 1) waren iterierte Kühlungen bei gleichem Gesamtkerngehalt verwendet worden. Wir hatten dabei angenommen, daß die damals erkannte Alterung der Gefrierkerne dafür sorgen würde, daß immer wieder andere, „schlechtere" Gefrierkerne zum Ansprechen kommen würden, so daß auf diese Weise ein allgemeines Spektrum trotz Beibehaltung derselben Tropfen resultieren würde. Diese Annahme konnte an unserem neuen, ungleich umfangreicheren Beobachtungsmaterial geprüft werden. Man brauchte nur jeweils die erste Kühlung jeder Versuchsreihe herauszugreifen, um verschiedene Kerne in voneinander ganz unabhängigen Tropfen zu haben. Das dafür bestimmte Spektrum, Kurve 20 (Abb.4), stimmt im großen ganzen

12 In neueren Versuchen haben wir die beschriebene gute Wirkung der verwendeten Substanzen auch erhalten, wenn wir den Kammerdeckel kurz abhoben und die Substanzen von außen auf die unterkühlten Tropfen aufstäubten. Die Temperaturbestimmung war dann allerdings nicht sehr genau möglich; auch eine evtl. Mitwirkung atmosphärischer Kerne wäre dann nicht ganz ausgeschlcssen. 
mit der Kurve 1 überein. Aber auch hier ist die Trennung der Wirkung der beiden Kernarten möglich: Kühlungen ohne Ventilation (Kurve 21) zeigen nur das zweite Maximum; mit Ventilation (Kurve 22) tritt aber schon eine beachtliche Anzahl Eisbildungen im ersten Maximum auf. Schon die Umwälzung der Luft in der Kammer kann also die Eisbildung beeinflussen: sie hat bei geschlossener Kammer frische Kerne in die Tropfen gebracht.

2. Daß diese Kerne aber nicht von dem (völlig gekapselten) Ventilator selbst stammen und daß sein Luftstrom auch nicht immer Kerne vorfindet, die er in die Tropfen befördern könnte, zeigt die Gruppe aller iterierten Kühlungen, denen schon mindestens eine Eisbildung vorangegangen war; Frischkernzufuhr war natürlich dabei unterblieben, weshalb auch die ersten Kühlungen der Versuchsreihen weggelassen wurden. In solchen Kühlungen kommt also nur die Alterung der Kerne zum Ausdruck und man erhält dann das ganz breite zweite Maximum der Kurve 23; Eisbildungen vorher sind sehr selten. Die Kurve 23 ist das direkte Gegenstück zur Kurve 19 (in Kurve 24 nochmals zum Vergleich eingezeichnet) für nur frische Kerne. Dabei war bei den Kühlungen zu 23 fast immer der Ventilator in Betrieb; es war aber durch Vermeidung größerer Temperaturunterschiede in der Kammer dafür gesorgt, daß, besonders beim Abschmelzen, an keiner Stelle der Kammer und auch nicht an den Tropfenrändern durch Abtrocknen neue aktive Kerne entstanden.

3. Das wichtigste Ergebnis liefert der Vergleich unserer Kurve 19 für nur frische Kerne mit der Kurve 24 für solche Kühlungen, die unmittelbar auf die Kühlungen der Kurve 19 folgten. Der Ventilator war dabei abgeschaltet, damit nicht etwa noch in der Kammer befindliche frische Kerne in die Tropfen gelangen konnten. Kurve 24 zeigt, daß von der Wirkung der guten Kerne in der darauffolgenden Kühlung gar nichts mehr zu merken ist. Die guten Kerne wirken also nur einmal, und obgleich sie sicher noch in den Tropfen enthalten sein müssen, gefrieren diese praktisch alle erst im zweiten Maximum. Aber schon wenn bei der folgenden Kühlung der Ventilator in Betrieb ist, erhält man wieder ganz deutlich beide Maxima (Kurve 25). In diesem Falle hat man noch in der Kammer befindliche Kerne mit Hilfe des Ventilators in die Tropfen gebracht.

4. Ein eigentlich selbstverständliches Ergebnis, das aber wegen der besonders instruktiven Kurvenform 26 (Abb. 4) angeführt werden soll, stellt sich ein, wenn man zu wenig Kerne in den Kernbehälter gegeben

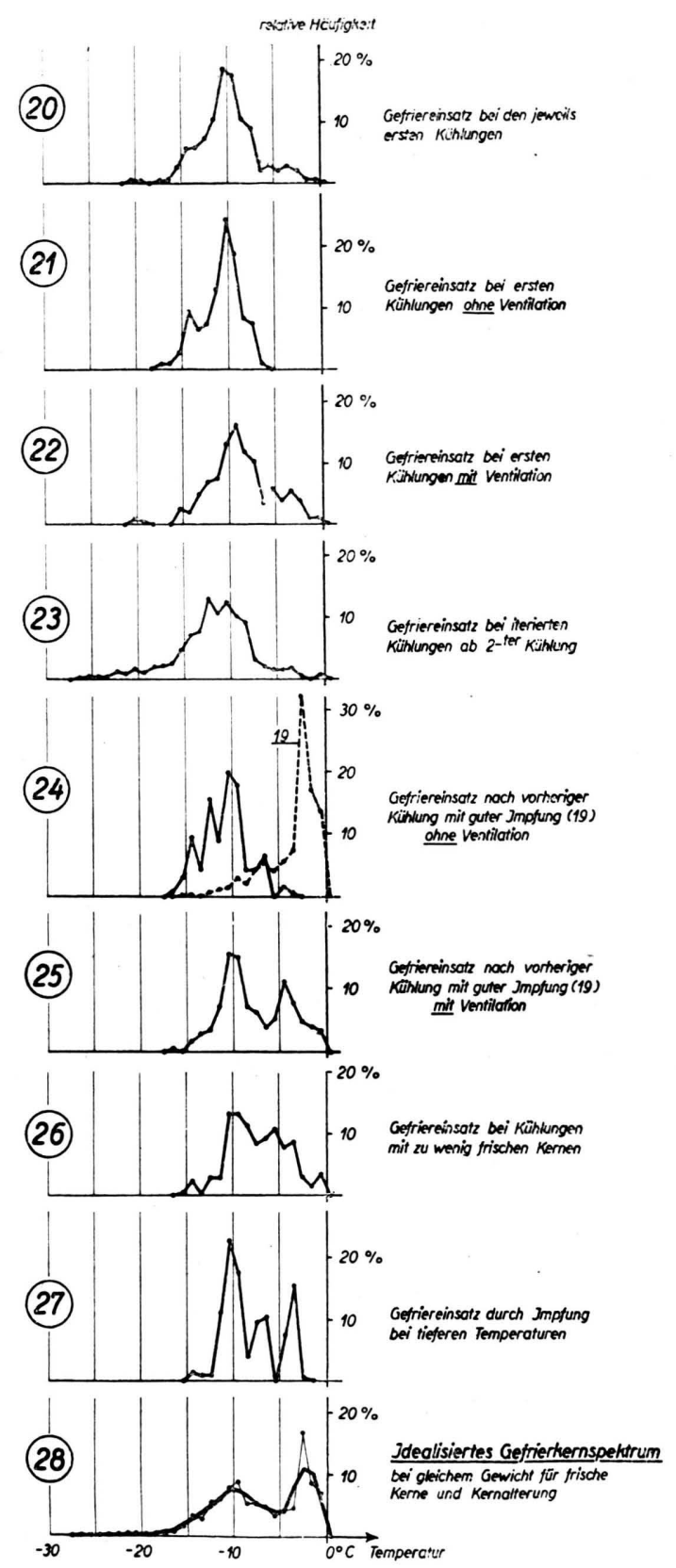

Abb. 4. Abhängigkeit des Gefrierkernsepektrums vom (jeweiligen) Kerngehalt.

hat. Der ganze Temperaturbereich ist dann gleichmäßig überdeckt und Häufungsstellen treten überhaupt nicht mehr auf. Die Impfung erfolgt dann langsamer und die Tropfen werden inzwischen immer kälter.

5. Hatten wir in Ziff. 3 dieses Abschnittes zeigen können, daß die für das erste Maximum verantwort- 
lichen Kerne in den Tropfen nur einmal wirken und schon bei der folgenden Kühlung keinen Effekt mehr geben, so gelang darüber hinaus die wesentliche Feststellung, daß diese Wirkung nur beim Eindringen der Kerne in bereits unterkühltes Wasser auftritt. Wurden die Substanzen noch bei Temperaturen über $0^{\circ} \mathrm{C}$ in die Tropfen gebracht, so blieben sie ohne Wirkung, und das auch, wenn es nur ganz kurz vor dem Passieren des Nullpunktes geschah.

Dagegen ist diese Wirkung aber nicht auf das Gebiet des ersten Maximums beschränkt, sondern die Kerne haben die „impfende“ Wirkung auch noch bei tieferen Temperaturen und man kann (analog Kurve 26) eine völlig fremde spektrale Verteilung (Kurve 27) hervorrufen, wenn man die Substanzen absichtlich erst bei stärkerer Unterkühlung (im vorliegenden Fall z. B. bei $-4,-6$ und $-10^{\circ} \mathrm{C}$ ) aufwirbelt.

Diese Wirkung der Kerne haben wir auch schon bei noch tieferen Temperaturen beobachtet; sie ist aber dort nicht mehr so deutlich von der Wirkung anderer Kerne (z. B. des zweiten Maximums) zu trennen.

6. Über die Wirkungsweise der Kerne im zweiten Maximum waren bei unseren Untersuchungen keine so wesentlichen Aussagen zu erhalten. Das kommt hauptsächlich daher, daß bei unseren Versuchsbedingungen der gewöhnliche Kerngehalt der Luft mitwirkt und die Eisbildung im Gebiet um -10 bis $-12^{\circ} \mathrm{C}$ so stark vorherrscht, daß tiefere Unterkühlungen, die zur Feststellung einer deutlichen Beeinflussung des zweiten Maximums notwendig gewesen wären, zu selten vorkamen. $\mathrm{Da}$ durch die Zugabe von frischem Kernmaterial aber auch das zweite Maximum beeinflußt wird, zeigt der Vergleich des dann sehr steil abfallenden zweiten Maximums der Kurve 5 mit dem breiteren und langsamer absinkenden Maximum der Kurve 4 für Kühlungen mit vorwiegend gleichbleibendem und nur alterndem Kerngehalt.

Im einzelnen wurde der Gefriereinsatz durch die aufgewirbelten Substanzen in der Folgekühlung ohne Ventilation in $10 \%$ der betreffenden Fälle deutlich heraufgesetzt, allerdings nur innerhalb des Temperaturbereiches des zweiten Maximums; in $10 \%$ der Fälle sank der Gefriereinsatz trotz der dazwischenliegenden Impfung sogar zu tieferen Temperaturen, während in $80 \%$ dieser Kühlungen die Eisbildung trotz der Impfung bei derselben Temperatur wie vorher wiederkam.

Man hat hier also eine Bestätigung der Tammann- schen Ergebnisse mit einer möglichen Erhöhung des Gefriereinsatzes, aber noch innerhalb des zweiten Maximums.

7. Wir haben damit zeigen können, daß die Eisbildung im unterkühlten Wasser durch Zugabe von als „Kernen“ wirkenden Fremdsubstanzen beeinflußbar ist. Die deutliche Wirkung beschränkte sich auf das erste Maximum, dessen Auftreten der Wirkung von frischen Kernen zuzuordnen war, die erst in das Wasser im unterkühlten Zustand gelangten. Durch Kerne, die schon im Tropfeninnern enthalten sind, bekommt man die Eisbildung offenbar immer erst im zweiten Maximum, auch wenn die Tropfen eine große Menge von Verunreinigungen enthalten. Zwischen diesen beiden Idealfällen sind alle Übergänge möglich, je nach Wahl der Versuchsbedingungen. Daß in den Spektren 1 und 2 gerade das zweite Maximum immer so stark hervortrat, bedeutet aber keineswegs, daß die im zweiten Maximum wirkenden Kerne in allen Fällen in so überwiegender Anzahl vorhanden wären. Dahinter verbirgt sich nur eine in unseren Versuchen zufällig eingetretene Bevorzugung von Kühlungen ohne Frischkerne, die zu den Kühlungen mit Frischkernen etwa im Verhältnis 3:1 standen. Errechnet man aus unseren experimentell ermittelten Häufigkeiten eine Verteilung für gleichviele Kühlungen mit frischen Kernen und Kühlungen mit gleichbleibendem, alterndem Kerngehalt, so führt diese Einführung gleichen statistischen Gewichts zu dem idealisierten $\mathrm{Ge}$ frierkernspektrum der Kurve 28, in dem sogar das erste Maximum noch leicht überwiegt.

8. In fast allen Fällen, wo in unseren Versuchen die Eisbildung zwischen 0 und etwa $-8^{\circ} \mathrm{C}$ erfolgte, konnten wir beobachten, daß sie durch Kerne ausgelöst wurde, die die Tropfen geimpft haben, wobei auch Kerne, die am Rand der Tropfen abgelagert sind, besonders wirksam waren. Wir glauben daraus schließen zu dürfen, daß überhaupt alle Eisbildungen in diesem Temperaturgebiet durch impfende Kerne erfolgen, und daß Kerne im Tropfeninnern erst im zweiten Maximum ansprechen. Dafür spricht auch die Tatsache, daß in fast allen Arbeiten über diesen Gegenstand eine Unterkühlung auf $-10^{\circ} \mathrm{C}$ in abgeschlossenen Gefäßen sehr leicht erreicht werden konnte, selbst bei beträchtlichen Verunreinigungen. $\mathrm{Da}$ dabei die im allgemeinen seltenen Eisbildungen oberhalb $-8^{\circ} \mathrm{C}$ meist auch bei vermutlich sehr trokkenen Kernsubstanzen eintraten, deutet darauf hin, daß dabei ebenfalls Impfung erfolgt ist, auch wenn dies, z. B. in Glasröhrchen, schlecht beobachtet werden konnte. 
Aus dieser absichtlich ausführlicheren Darstellung ist zu ersehen, wie stark die Vorgeschichte des untersuchten Kerngehaltes den Gefriereinsatz im unterkühlten Wasser beeinflußt und welche Schwierigkeiten in der Deutung der Beobachtungen auftreten können, sobald die Versuchsbedingungen nicht einwandfrei zwischen den Wirkungen der verschiedenen Kernarten unterscheiden lassen, vor allem dort, wo auch in abgeschlossenen Räumen der einmal vorhandene Kerngehalt infolge von Temperaturschwankungen doch nicht gleich aktiv bleiben wird. Die Folge davon ist dann die immer wieder betonte schlechte Reproduzierbarkeit der Unterkühlungsversuche bei Wasser.

\section{Zur Deutung der Wirkungsweise der Gefrierkerne}

Wenn man nicht gerade annehmen will, daß bei den von uns untersuchten Substanzen immer eine unbekannte Komponente dabei ist, die die Eisbildung auslöst ${ }^{13}$, so ist aus unseren Beobachtungen zu schließen, daß man es bei der Wirkung der Kerne im ersten Maximum mit einer ganz allgemeinen, von der speziellen Struktur unabhängigen Erscheinung zu tun haben muß. Gegen eine solche Komponente spricht aber schon die entscheidende Bedeutung der Trockenheit der Kerne und die Beobachtung, daß die Kerne nur im Augenblick des Eintauchens in unterkühltes Wasser dessen Kristallisation auslösen. Wie schon erwähnt, wurde zunächst vermutet, daß sich die Kerne vielleicht beim Aufwirbeln elektrisch aufladen. Aber es gelang nicht, sie durch ein elektrisches Feld am Erreichen der Tropfen zu hindern. Daß die Kerne aber nur so gut wirken, wenn sie in die Tropfen eindringen, erinnert an den Grundversuch zur Entstehung der Reibungs-, besser der Berührungselektrizität nach $\mathrm{Pohl}^{14}$, wo eine Paraffinkugel, an isoliertem Stiel in Wasser eingetaucht, sich negativ auflädt, das Wasser aber positiv geladen wird. Die Ursache der Aufladung ist darin zu sehen, daß sich in der Grenzschicht zweier Phasen ein starkes elektrisches Feld, allerdings nur auf Entfernungen der Größenordnung von Molekülabständen $\left(10^{-8} \mathrm{~cm}\right)$ ausbildet: die sog. Doppelschicht. Diese ist besonders aus den Erscheinungen der Elektrokinese bekannt, wo sie sich an Quarzteilchen, Metallteilchen, Öltröpfchen u. a. bildet.

13 Diese Annahme ist natürlich bei den erwähnten Versuchen anderer Autoren genau so möglich.

14 R. W. P o h l, Einführung in die Elektrizitätslehre, 10. u. 11. Aufl., S. 207 [1944].
Wir möchten nun die beobachtete Impfung so deuten, daß sich bei unseren Kernen beim Eintauchen in das Wasser diese Doppelschicht bildet; die damit verbundene Orientierung der Wassermoleküle (Dipole) bedeutet eine Behinderung der freien Molekularbewegung, so daß diese die Keimbildung nicht mehr wie im freien Wasser verhindert; da die Zustandsbedingungen im unterkühlten Wasser schon Eiswachstum gestatten, gefriert der Tropfen. Geht der Kern aber schon bei Temperaturen über $0^{\circ} \mathrm{C}$ in den Tropfen, so bildet sich die Doppelschicht bereits aus, solange die Wachstumsbedingungen für die Eisphase noch gar nicht erfüllt sind: der Tropfen kann nicht erstarren, und wenn die Temperatur später unter $0^{\circ} \mathrm{C}$ absinkt, so ist der Effekt der Doppelschichtbildung schon abgeschlossen. Auch der Abtrocknungseffekt der Kerne wäre zwanglos einzureihen. Nur ein trockener Kern kann bei dieser Deutung der Vorgänge keimbildend wirken; ein feuchter Kern wirkt als kleines Tröpfchen; beim Eintauchen in Wasser kommen dann gar keine verschiedenen Phasen mehr in Berührung; die impfende Wirkung bleibt aus.

Es bliebe noch zu ergänzen, daß nach unseren Beobachtungen bei der Impfung offenbar auch die Kerngröße eine Rolle spielt, sei es, daß von ihr die Möglichkeit abhängt, daß der Kern überhaupt in den Tropfen eindringen kann, sei es, daß die Feldstärke in der Doppelschicht damit zusammenhängt. Wenn man bedenkt, wie nach der Th o m p s o n schen Formel der Dampfdruck über Tröpfchen ab $1 \mu$ Größe anfängt, merklich größer zu werden als über der ebenen Phase, so erscheint eine solche Einwirkung der Kerngröße verständlich; hat man es doch letzten Endes in beiden Fällen mit denselben molekularen Kräften zu tun.

Unser Deutungsversuch wird gestützt durch neuere Versuche, wo wir durch hohe elektrische Felder im Wasser eine (offenbar ohne Kerne stattfindende) Kristallisation auslösen konnten. Darüber soll in einer späteren Arbeit berichtet werden.

VII. Folgerungen für die atmosphärische Eisbildung

Wie wir in der früheren Arbeit ${ }^{7}$ gezeigt haben, erhält man volle Úbereinstimmung zwischen unseren experimentellen Häufigkeitsverteilungen für bestimmte Unterkühlungen im Laboratorium mit den von $\mathrm{P}$ ep ple ${ }^{15}$ für die atmosphärischen Unterküh-

15 W. P e p ple r, Z. angew. Meteor. 56, 173 [1939] u. Forschg. Erfahr.-ber. Reichswetterdienst, Reihe B, N 1 [1940]. 
lungen festgestellten Häufigkeitsverteilungen, wenn man aus unseren Gefrierkernspektren als den Verteilungskurven für die Temperatur des Gefriereinsatzes die „integralen“ Kurven für die Häufigkeit von Unterkühlungen bis zu bestimmten Temperaturen errechnet.

In der Atmosphäre erfolgt die Eisbildung nach Peppler hauptsächlich um $-12^{\circ} \mathrm{C}$, also in unserem zweiten Maximum. Aus dem konformen Verlauf seiner damit eigentlich nicht verträglichen Kurve der atmosphärischen Unterkühlungen - aus dem Vereisungsbeginn am Flugzeug - mit unserer integralen Kurve für genügend frische Kerne (zu Kurve 19) haben wir damals geschlossen, daß unter gewissen Bedingungen eigentlich auch um $-4^{\circ} \mathrm{C}$ in der Atmosphäre eine bevorzugte Eisteilchenbildung vorkommen müßte. Inzwischen hat $\mathrm{S} \mathrm{chwerd} \mathrm{tf} \mathrm{e} \mathrm{g} \mathrm{e}{ }^{16}$ nachweisen können, daß bei der Schauerbildung über See zumindest im Sommer, in einer erheblichen An-

16 W. S c hwerdtf e g e r, Meteor. Rundschau 1, 453 [1948]. zahl von Fällen aber auch in den übrigen Jahreszeiten, der $-12^{\circ}$-Bereich gar keine bevorzugte Rolle spielt, wogegen gerade der Bereich um $-4^{\circ} \mathrm{C}$ mit bemerkenswerter Häufigkeit hervortritt. Schwerdtfegers Darstellung, daß eine entscheidende Wirkung der Gefrierkerne erst bei $-12^{\circ} \mathrm{C}$ angenommen würde, bedarf allerdings nach unseren Ergebnissen einer Richtigstellung dahingehend, daß die Wirksamkeit der Gefrierkerne von uns experimentell ermittelt worden ist und schon das früher ${ }^{6}$ veröffentlichte $\mathrm{Ge}$ frierkernspektrum die beiden Maxima bei $-4^{\circ} \mathrm{C}$ und um $-12^{\circ} \mathrm{C}$ zeigte. Eine Bevorzugung des ersten Maximums bei der Schauerbildung bedeutet nach unseren neuen Ergebnissen die vorwiegende Wirkung von frischen, abgetrockneten Kernen (gemäß unserer Kurve 19), wobei sogar an eine Impfung durch Salzteilchen (gemäß unserer Kurve 10 a) zu denken wäre.

Die beobachtete Wirkung der Kerne im ersten Maximum macht es auch verständlich, daß in der Natur größere Wassermengen nie merklich unterkühlt sind.

\title{
Zur Relaxation und Assoziation von polaren Kettenmolekülen in der reinen Flüssigkeit
}

\author{
Von G. Klages und G. Kremmling \\ Aus dem Physikalischen Institut der Johannes-Gutenberg-Universität Mainz \\ (Z. Naturforschg. 5 a, 675-682 [1951]; eingegangen am 13. Dezember 1950)
}

\begin{abstract}
Absorptions- und Dispersionsmessungen im Gebiet von 10 bis $300 \mathrm{~m}$ an langkettigen aliphatischen Chloriden und Alkoholen in der reinen Flüssigkeit werden nach der Debye-Rammschen Theorie der Rotationsbehinderung und der Onsagerschen Theorie diskutiert und mit Messungen in der verdünnten Lösung verglichen. Die nichtassoziierenden Chloride zeigen eine größere Dipolbeweglichkeit in der reinen Flüssigkeit, die auf eine besondere Flüssigkeitsstruktur (Parallellagerung der Ketten) zurückgeführt wird. Bei den Alkoholen sinkt die Beweglichkeit durch Assoziation, die mit zunehmender Kettenlänge monoton abnimmt. Die exakte Ermittlung des Assoziationsgrades macht noch Schwierigkeiten, da bei den langkettigen Alkoholen noch eine Dipolbeweglichkeit innerhalb der Komplexe vorhanden ist, was aus dem angedeuteten Auftreten von mehreren Relaxationszeiten und Dipolabsorption im glasigen und kristallinen Zustand geschlossen wird.
\end{abstract}

$\mathrm{D}_{\mathrm{in}}^{\mathrm{i}}$

e Größe der Relaxationszeit von Dipolmolekülen in der Flüssigkeit, die bestimmend ist für die Lage des Gebiets der Dipolverluste und anomalen Dispersion im elektromagnetischen Spektrum ${ }^{1}$, wird durch mehrere Faktoren bedingt. Neben der Größe und Form des Moleküls, seiner inneren Beweglichkeit und der Lokalisierung des Dipols spielen die Wechselwirkungskräfte des Moleküls mit seiner Um-

1 P. Deb y e, Polare Molekeln, S. Hirzel, Leipzig 1929. gebung eine entscheidende Rolle. Sie können in erster Näherung - besonders bei verdünnten Lösungen in einem unpolaren Lösungsmittel - durch eine Reibungsgröße erfaßt werden, die der makroskopisch gemessenen Viskosität $\eta$ proportional ist. In der reinen Flüssigkeit ruft die Wechselwirkung eines Dipolmoleküls mit den Nachbardipolen die Ausbildung einer gewissen Orientierung der Dipole hervor, aus der zusätzliche Wechselwirkungskräfte resultieren. 